\title{
54 DEVELOPMENT, IMPLEMENTATION AND EVALUATION OF LOW SPEED VEHICLE RUNOVER EDUCATION INTERVENTION
}

doi:10.1136/injuryprev-2012-040590d.54

KA Bron, Include Susan Teerds Roy.

Background LSVRO incidents are a significant cause of paediatric transport-related morbidity and mortality in Queensland-there are approximately three LSVRO incidents per week. Together with Kidsafe Queensland, an intervention (poster/sticker) was designed based on preliminary research findings of a retrospective analyses of 11 years of linked date on LSVRO incidents in OLD.

Aims This study was designed to evaluate the impact of a population-level education intervention to increase awareness regarding LSVRO incidents, and also to identify relevant target groups with low knowledge or inadequate behaviour in relation to LSVRO incidents.

Method Kidsafe Queensland coordinated the poster printing and distribution. The poster was sent to all early childhood education centres, kindergartens, maternal child health units, and Kidsafe House. Prior to the intervention implementation, a cross-sectional sample of the population was interviewed at a shopping centre (pre-intervention), and this was repeated 4-months post-intervention. Data collected included: Demographic characteristics; LSVRO Environmental Risk Factors; LSVRO Knowledge, Attitudes and Behaviour.

Results Participants in the study had poor knowledge regarding LSVRO incidents, and poor safe driveway behaviour. The results highlight that there is no one group to target in relation to increasing awareness regarding LSVRO incidents, and prevention. There were limitations to this study, but the findings provide some evidence that the population-level intervention was successful in increasing awareness and improving behaviour regarding LSVRO incidents.

Significance/Contribution to the Field This is the first survey known to the authors that has assessed knowledge, attitudes and behaviour in relation to LSVRO incidents. 